\title{
Bacopa monnieri Extract (CDRI-08) Modulates the NMDA Receptor Subunits and nNOS-Apoptosis Axis in Cerebellum of Hepatic Encephalopathy Rats
}

\author{
Papia Mondal and Surendra Kumar Trigun \\ Biochemistry Section, Department of Zoology, Banaras Hindu University, Varanasi 221005, India \\ Correspondence should be addressed to Surendra Kumar Trigun; sktrigun@gmail.com
}

Received 27 November 2014; Revised 9 February 2015; Accepted 9 February 2015

Academic Editor: Con Stough

Copyright (C) 2015 P. Mondal and S. K. Trigun. This is an open access article distributed under the Creative Commons Attribution License, which permits unrestricted use, distribution, and reproduction in any medium, provided the original work is properly cited.

\begin{abstract}
Hepatic encephalopathy (HE), characterized by impaired cerebellar functions during chronic liver failure (CLF), involves Nmethyl-D-aspartate receptor (NMDAR) overactivation in the brain cells. Bacopa monnieri (BM) extract is a known neuroprotectant. The present paper evaluates whether BM extract is able to modulate the two NMDAR subunits (NR2A and NR2B) and its downstream mediators in cerebellum of rats with chronic liver failure (CLF), induced by administration of $50 \mathrm{mg} / \mathrm{kg}$ bw thioacetamide (TAA) i.p. for 14 days, and in the TAA group rats orally treated with $200 \mathrm{mg} / \mathrm{kg}$ bw BM extract from days 8 to 14. NR2A is known to impart neuroprotection and that of NR2B induces neuronal death during NMDAR activation. Neuronal nitric oxide synthase- (nNOS-) apoptosis pathway is known to mediate NMDAR led excitotoxicity. The level of NR2A was found to be significantly reduced with a concomitant increase of NR2B in cerebellum of the CLF rats. This was consistent with significantly enhanced nNOS expression, nitric oxide level, and reduced Bcl2/Bax ratio. Moreover, treatment with BM extract reversed the NR2A/NR2B ratio and also normalized the levels of nNOS-apoptotic factors in cerebellum of those rats. The findings suggest modulation of NR2A and NR2B expression by BM extract to prevent neurochemical alterations associated with HE.
\end{abstract}

\section{Introduction}

The patients with liver cirrhosis develop a serious nervous system disorder known as hepatic encephalopathy (HE) [1]. It is characterized by wide spectrum of neuropsychiatric symptoms related to motor dysfunction, cognitive impairment, and disturbed sleep wake cycle [2-4]. Most of the liver cirrhotic patients have been found to show minimal to overt HE symptoms $[5,6]$, characterized mainly by the impaired motor functions [2,7-9], which is considered to be associated with deranged cerebellar functions $[10,11]$. Some recent findings from our lab also suggest that cerebellum shows greater susceptibility to undergo neurochemical changes in the models of chronic type HE [12-14]. Therefore, cerebellum was selected for the present study.

Based on the studies conducted to understand pathophysiology of HE, it has been suggested that the level of glutamate, an important excitatory neurotransmitter, increases in the synaptic cleft due to the increased blood and brain ammonia level during liver dysfunction, resulting into overactivation of the ionotropic N-methyl-D-aspartate receptors (NMDAR) [1]. And there is a general agreement that activation of glutamate-NMDA receptor-nNOS pathway constitutes main neurochemical aberrations associated with HE $[1,14]$.

Functional NMDAR, a tetrameric protein complex, comprises of two subunits of a constitutive glycine binding NR1 and remaining two of glutamate binding NR2 from amongst NR2A, NR2B, NR2C, and NR2D subunits. Importantly, combination of different NR2 subunits is suggested to confer unique electrophysiological properties to this neurotransmitter receptor $[15,16]$. For example, alterations in the ratio of NR2A versus NR2B of NMDAR have been found to be associated with the changes in long term potentiation (LTP) and long term depression (LDP) functions during hippocampal plasticity involved in memory consolidation [17]. In addition, NR2A dominating combination of NMDAR is demonstrated to provide neuroprotection but NR2B rich 
NMDAR is known to drive the postsynaptic neuron towards apoptosis during glutamate excitotoxicity [14]. Thus, regulating NMDAR function by altering its composition, without pharmacological blockage of the channel, could be a unique and novel cerebral mechanism to prevent NMDAR overactivation led neurological disorders.

It is now evident that neuronal nitric oxide synthase (nNOS), via modulating NO level in the brain cells, plays critical roles in transmitting NMDAR led neurophysiological changes under different pathophysiological conditions [18, 19]. A threshold level of $\mathrm{NO}$ is essential to activate NOcGMP signaling to maintain NMDAR dependent memory consolidation and cognition functions [18]. However, excess of NO is known to induce apoptosis and neuronal death [20, 21]. Such multimodal roles of NMDAR-nNOS axis in brain cells are orchestrated by a molecular link between NMDAR and nNOS protein. The NR2 subunits of NMDAR complex, through their tSXV motifs, connect with the postsynaptic density protein-95 (PSD-95), which in turn, via its PDZ domain, interacts with the nNOS in the postsynaptic neurons $[19,22]$. This constitutes the main mechanism of NMDAR activity led NO production and subsequent changes in the neuronal functions. Particularly during $\mathrm{HE}$, activation of NMDAR leads to the increased calcium influx which in turn activates nNOS and thereby overproduces NO in the postsynaptic neurons $[1,9,13]$. Thus increased glutamate led NMDAR activation, via activation of nNOS, is considered critically involved in developing $\mathrm{HE}$ associated neuropsychiatric problems in the patients/animals $[13,23,24]$.

Obviously NMDAR becomes choice of an important therapeutic target for the neurobiologists for HE management $[25,26]$. Some earlier studies conducted in vivo and in vitro using NMDAR antagonists indeed demonstrated desirable results; however, this approach was found to produce undesirable neurological complications during the clinical trials $[25,27]$. This is not surprising as NMDAR activity is critical for maintaining normal neurophysiology including higher order brain functions and memory consolidation mechanisms [17]. Therefore, instead of blocking NMDAR channel, modulation of NMDAR activity by alterations in its functional composition and downstream signaling seems to be of special scientific merit. However, this evolving concept needs to be examined in the animal models with excitotoxic neurological problems. Since development of $\mathrm{HE}$ is related with NMDAR led excitotoxicity [1] and that herbal formulations are now evident to modulate brain chemistry in many ways, the present work was undertaken to evaluate whether Bacopa monnieri (BM) extract, a known neuroprotectant, is able to modulate NMDAR composition and related downstream events in cerebellum of the CLF induced HE rats.

Amongst a good number of herbal drugs available, Bacopa monnieri extract has been widely evaluated as a memory enhancer, adaptogenic, anti-inflammatory, analgesic, antipyretic, sedative, and antiepileptic agent [28]. Also, some studies suggest its neuroprotective roles against epilepsy (neuroexcitotoxic outcome) by modulating serotonergic receptor [29], and against Parkinson's and Alzheimer's disease via altering dopaminergic signaling [30] and cholinergic [31] receptors, respectively. Moreover, molecular mechanisms underlying these BM extract effects remain largely unexplored.

Importantly, though information is limited, efficacy of BM extract has also been shown against glutamate toxicity via modulating NMDAR1 gene expression and in turn affecting glutamatergic signaling [32]. In our previous reports, we have observed a direct association between overexpression of the constitutive NR1, nNOS activation, and enhanced NO production in cerebellum of the CLF rats exhibiting $\mathrm{HE}$ characteristics $[2,13,14]$. Importantly, we could also observe reciprocal expression of NR2A and NR2B in the cerebellum of those rats (data from this paper). This tempted us to investigate whether administration of BME is able to alter this unusual NR2A/2B composition and thus NMDAR-nNOS pathway in the cerebellum of the HE rats.

\section{Materials and Methods}

2.1. Chemicals. Chemicals used were of analytical grade supplied by E-Merck and Sisco Research Laboratory (India). Acrylamide, $N, N^{\prime}$-methylenebisacrylamide, N,N, $\mathrm{N}^{\prime} \mathrm{N}^{\prime}$-tetramethylethylenediamine (TEMED), phenyl methyl sulphonyl fluoride (PMSF), bromophenol blue, and Ponceau were purchased from Sigma-Aldrich, USA. Primary antibodies used were procured from the following companies: rabbit monoclonal $\beta$-actin from Sigma Aldrich, rabbit monoclonal anti-NR2B from Invitrogen, rabbit monoclonal NR2A from Epitomics, rabbit polyclonal $\mathrm{Bcl} 2$ and $\mathrm{Bax}$ from Cell Signaling Technology, and rabbit polyclonal nNOS from Santa Cruz Biotechnology. Rabbit and mouse horseradish peroxidase (HRP) conjugated secondary antibodies were obtained from Genei. ECL western blotting detection kit was purchased from Thermo Scientific.

The ethanolic extract of Bacopa monnieri extract (BM/ CDRI-08), containing $64.28 \%$ bacoside A and $27.11 \%$ bacoside B, was obtained from the Lumen Research Foundation, Chennai, India.

2.2. Animals. Inbred adult female albino rats weighing 150$160 \mathrm{~g}$ were used in this study. The rats were kept in separate cages, fed with the recommended diet, and maintained at standard conditions of $12 \mathrm{~h}$ light and dark period at room temperature $\left(25 \pm 2^{\circ} \mathrm{C}\right)$ in an animal house. The use of animals for the present study was approved by the Institutional Animal Care and Use Committee (IACUC); Animal Ethical Committee (AEC) of the Banaras Hindu University, Varanasi.

\subsection{Induction of Chronic Liver Failure (CLF)/HE and Treat-} ment Schedule. The CLF/HE model of neuroexcitotoxicity in adult albino rats was induced by the administration of thioacetamide (TAA) as standardized previously [2]. For this, rats were randomly divided into three groups with 6 rats in each. Group A: control (C), administered with 0.9\% saline i.p, once daily for 14 days; Group B: CLF/HE group, administered with $50 \mathrm{mg} / \mathrm{Kg}$ bw TAA i.p once daily for 14 days; Group C: CLF + Bacopa extract (CLF + BM). Rats in Group $\mathrm{C}$ were orally administered with ethanolic extract of BM extract (CDRI-08; $200 \mathrm{mg} / \mathrm{Kg} \mathrm{bw}$ ), suspended in $1 \%$ 
gum acacia, once daily starting from 8th day onwards till 14 th day, and were administered $4 \mathrm{~h}$ after the TAA treatment. The dose of BM was selected which was able to recover TAA induced neurobehavioural deficit in the rats. All the rats were sacrificed on 15th day. The cerebellum was dissected out and stored at $-80^{\circ} \mathrm{C}$ for further experiments.

2.4. Preparation of Cerebellar Extracts. As described earlier [13], mitochondria free cerebellar extracts were prepared in an extraction medium consisting of $400 \mathrm{mM}$ sucrose, $1 \mathrm{mM}$ EDTA, $0.2 \mathrm{mM}$ benzamidine, $0.1 \mathrm{mM}$ phenylmethylsulfonyl fluoride (PMSF), and $0.02 \%$ heparin. The extracts were centrifuged initially at $12000 \times \mathrm{g}$ for $15 \mathrm{~min}$ and finally at $19000 \times \mathrm{g}$ for $45 \mathrm{~min}$ at $4^{\circ} \mathrm{C}$. The supernatant obtained was collected as the cytosolic fractions. The protein content in the tissue extract was measured by Lowry method [33] using BSA as standard.

2.5. Western Blotting. As described previously [13], the cytosolic fractions containing $60 \mu \mathrm{g}$ protein/lane were separated on $10 \%$ SDS-PAGE and electrotransferred to nitrocellulose membrane at $50 \mathrm{~mA}$ and run overnight at $4^{\circ} \mathrm{C}$. Protein transfer was checked via Ponceau staining. The membrane was then placed in a blocking solution of 5\% skimmed milk in $1 \mathrm{X}$ PBS for $2 \mathrm{~h}$ followed by washing in PBS 3 times. The membranes were then separately processed for immunodetection of NR2A, NR2B, nNOS, Bcl2, and Bax using monoclonal/polyclonal anti-NR2A (1:1000), anti-NR2B (1:1000), anti-nNOS (1:500), anti-Bcl2 (1:1000), and anti-Bax (1:500), respectively. HRP conjugated secondary antibody was used for final detection of the proteins using ECL western blotting detection kit. $\beta$-actin, used as loading control, was detected using a monoclonal anti- $\beta$-actin peroxidase antibody. The bands were quantified and analyzed using gel densitometry software AlphaImager 2200. The photographs in the figure are representatives of the three western blot repeats.

2.6. Nitric Oxide (NO) Estimation. Nitric oxide level was measured by estimating total nitrite $\left(\mathrm{NO}_{2}\right)$ and nitrate $\left(\mathrm{NO}_{3}\right)$ content in the tissue extracts as described earlier [13] using the method of Sastry et al. [34]. Briefly, tissue fractions $(100 \mu \mathrm{L}), \mathrm{NaNO}_{2}$, and $\mathrm{KNO}_{3}$ standards $(0.1 \mathrm{mM}$ each) were mixed separately with $400 \mu \mathrm{L}$ of $50 \mathrm{mM}$ carbonate buffer ( $\mathrm{pH}$ 9). For $\mathrm{NO}_{3}$ estimation, activated copper-cadmium alloy $(150 \mathrm{mg})$ was added and incubated for an hour at $37^{\circ} \mathrm{C}$. The reaction was stopped using $100 \mu \mathrm{L}$ each of $0.35 \mathrm{M} \mathrm{NaOH}$ and $120 \mathrm{mM}$ zinc sulphate. After centrifugation, $400 \mu \mathrm{L}$ supernatant was incubated with the Griess reagent: $200 \mu \mathrm{L} 1 \%$ sulphanilamide prepared in $2.5 \% \mathrm{H}_{3} \mathrm{PO}_{4}$ and $200 \mu \mathrm{L} 0.1 \% \mathrm{~N}$ (1-naphthyl)-ethylenediamine. Absorbance was recorded at $545 \mathrm{~nm}$. For $\mathrm{NO}_{2}$ estimation, similar procedure was followed except addition of the copper-cadmium alloy.

2.7. Statistical Analysis. The data have been expressed as mean \pm SD. For two group comparisons, statistical analysis was performed using unpaired Student's $t$ test. A probability of $P<0.05$ was taken as a significant difference between the groups. Each of the experiments was repeated thrice.

\section{Results}

3.1. Effect of BM Extract on NR2A and NR2B Expression. The combination of constituent NMDAR subunits is evident to impart unique neurophysiological role of this glutamate receptor. The NR2A dominating combination is known to mediate neuroprotection whereas those of NR2B induce neuronal death. According to Figures 1(a) and 1(b), as compared to the control rats, level of NR2A is found to be significantly reduced $(P<0.001)$ with a concomitant increase in NR2B level in the cerebellum of the CLF rats (Figures 1(c) and $1(\mathrm{~d})$ ), resulting in a significant decline in NR2A/2B ratio (Figure 1(e)). However, this pattern is observed to be recovered back with a significant enhancement of NR2A (Figures 1(a) and 1(b)) and a decline of NR2B (Figures 1(c) and $1(\mathrm{~d})$ ), resulting into attaining a control level NR2A/2B ratio (Figure $1(\mathrm{e})$ ) in the cerebellum of the CLF rats treated with the BM extract.

\subsection{Effect of BM Extract on nNOS and Nitric Oxide (NO)} Production. Neuronal NOS (nNOS) has a direct molecular link with NMDAR and therefore, it is considered to be the main determinant of NMDAR activation based downstream signaling in the postsynaptic neurons. Accordingly, overactivation of nNOS is considered associated with the neuronal changes associated with NMDAR led excitotoxicity. As depicted in Figures 2(a) and 2(b), nNOS expression is observed to be enhanced significantly $(P<0.001)$ in the cerebellum of the CLF rats as compared to the control group rats. Moreover, due to the oral administration of BM extract, level of this enzyme is observed to be reduced up to the control value (Figures 2(a) and 2(b)). Such a pattern could coincide well with the similar changes in the NO level in cerebellum of the CLF and BM extract treated CLF rats (Figure 2(c)).

3.3. Effect of BM Extract on the Expression of Bcl2 and Bax. $\mathrm{Bcl} 2$ (antiapoptotic) and Bax (proapoptotic) ratio serves as a rheostat to determine cell susceptibility to apoptosis. According to Figures 3(a) and 3(c), as compared to the control group rats, level of $\mathrm{Bcl} 2$ is found to be declined significantly $(P<0.05)$ with a concomitant increase in the Bax level resulting in a significant decline $(P<0.01)$ in $\mathrm{Bcl} 2 / \mathrm{Bax}$ ratio (Figure 3(e)) in cerebellum of the CLF rats. However, after treatment with $\mathrm{BM}$ extract, the pattern of $\mathrm{Bcl} 2 / \mathrm{Bax}$ ratio is observed to regain its normal range in cerebellum of those CLF rats (Figure 3(e)).

\section{Discussion}

NMDA receptor overactivation is considered a common neurochemical event associated with a number of brain dysfunctions like epilepsy, ischemia, drug abuse, and $\mathrm{HE}$ [23, 26, 35-37]. However, NMDAR blockage is evident to be a poor therapeutic target for managing such excitotoxic conditions $[25,27]$. In this background, while understanding neurochemical basis of CLF induced HE, we observed a clear 


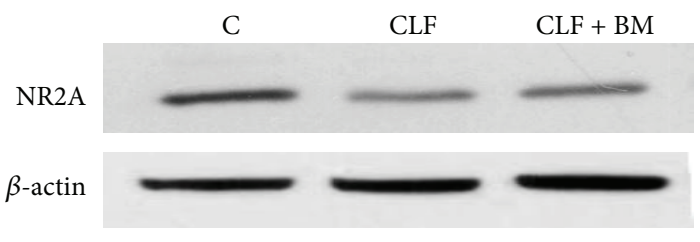

(a)

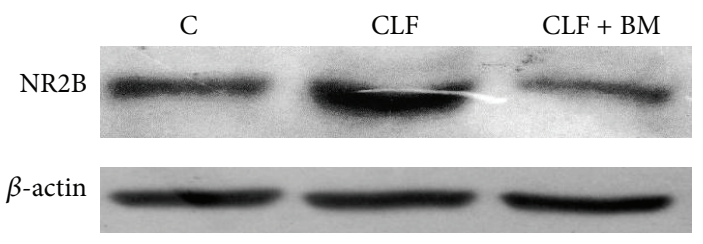

(c)

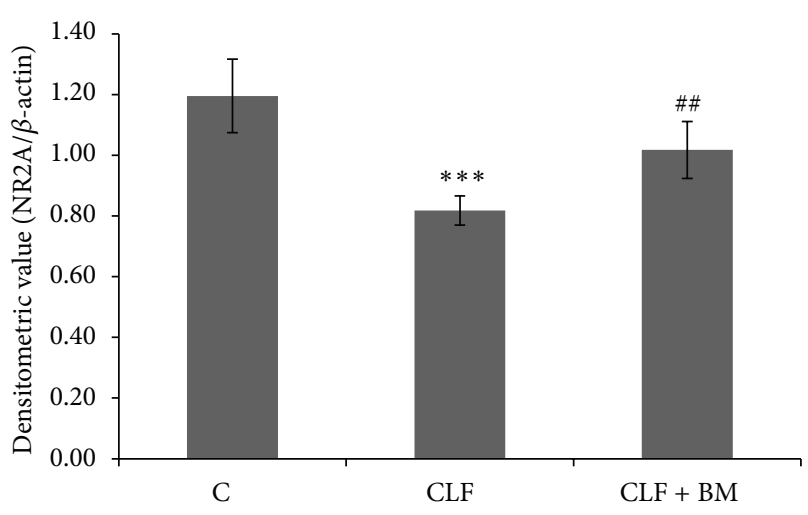

(b)

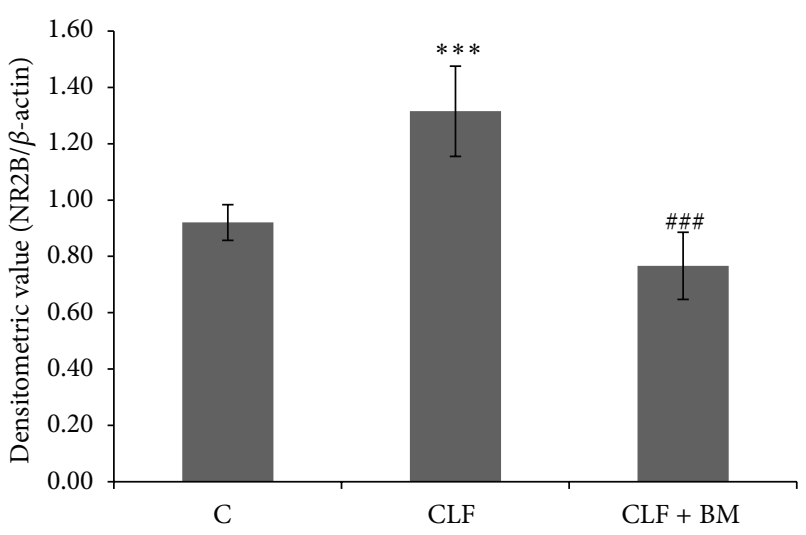

(d)

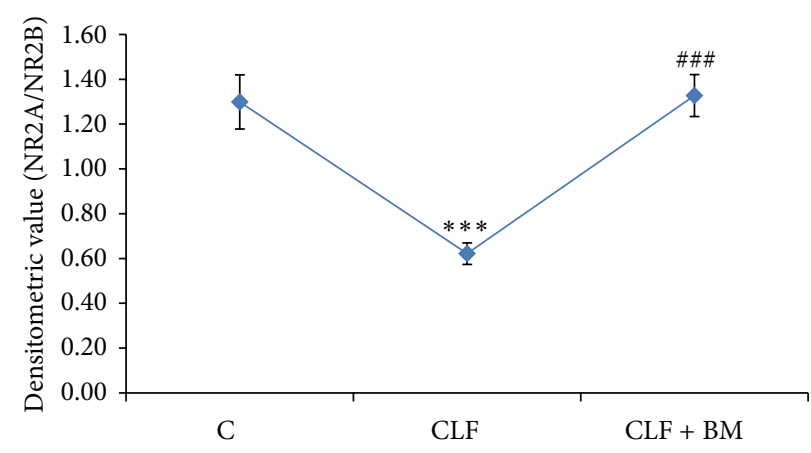

(e)

FIGURE 1: Expression profile of NR2A ((a) and (b)) and NR2B ((c) and (d)) in cerebellum of the CLF and BM extract treated CLF rats. Western blot analysis was performed as described in the method text. The level of $\beta$-actin was probed as the loading control. In panels (b), (d), and (e), normalized densitometric values of NR2A/ $\beta$-actin, NR2B/ $\beta$-actin, and NR2A/NR2B have been presented as mean \pm SD from three western blot repeats. ${ }^{* * *} P<0.001$; (control versus CLF rats) ${ }^{\# \#} P<0.01,{ }^{\# \# \#} P<0.001$; (CLF versus CLF + BM rats). C: control, CLF: chronic liver failure, CLF + BM: chronic liver failure + Bacopa monnieri extract.

shift in the expression of glutamate binding NMDAR subunit from a NR2A dominating combination to a NR2B rich combination in cerebellum of the CLF rats (Figures $1(\mathrm{a})$ and $1(\mathrm{c})$ ). It is now becoming clearer that NR2A rich NMDAR imparts neuroprotection and that of NR2B dominating combination initiates neuronal damage and apoptosis $[16,38-40]$. This is because NR2B has been reported to show delay in gating kinetics in comparison to that of NR1/NR2A combination resulting in increased $\mathrm{Ca}^{2+}$ influx and thus rapid activation of the downstream signaling [41]. In the present context, a significant decline in Bcl2 with a concomitant increase in Bax level in cerebellum of the CLF rats (Figure 3) also hints for a direct association between NR2B dominating NMDAR composition and altered neurochemistry of cerebellum of the CLF rats. Furthermore, alterations in oxidative and nitrosative factors in the postsynaptic neurons are considered to be the main downstream mediators of such unusual NMDAR activations [1] and it has been reported that cerebellum shows 


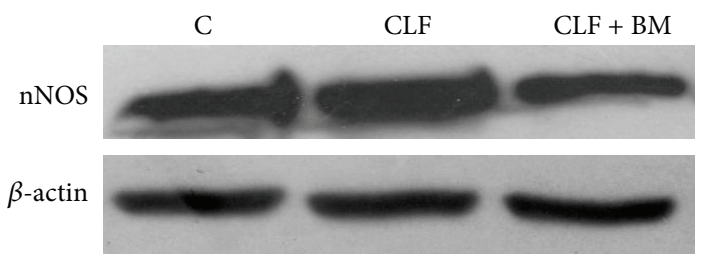

(a)

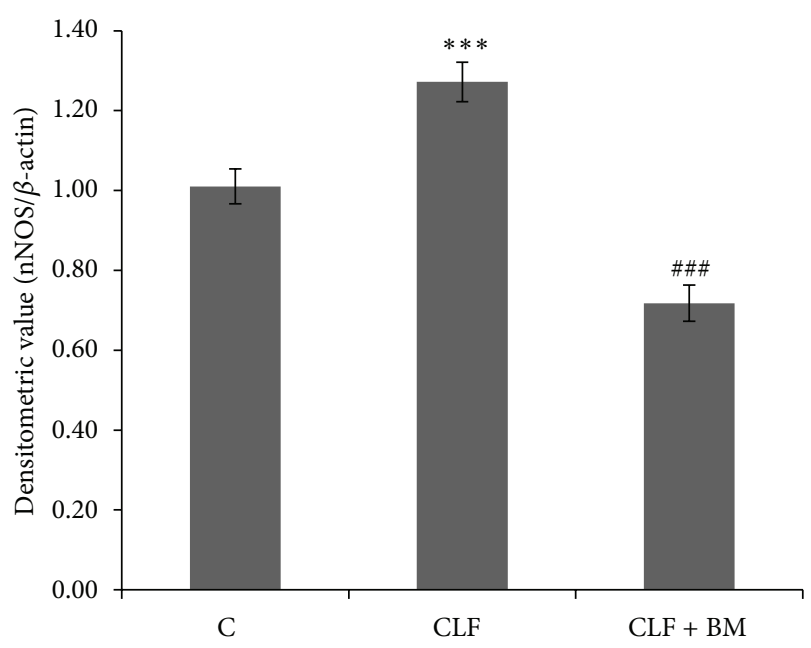

(b)

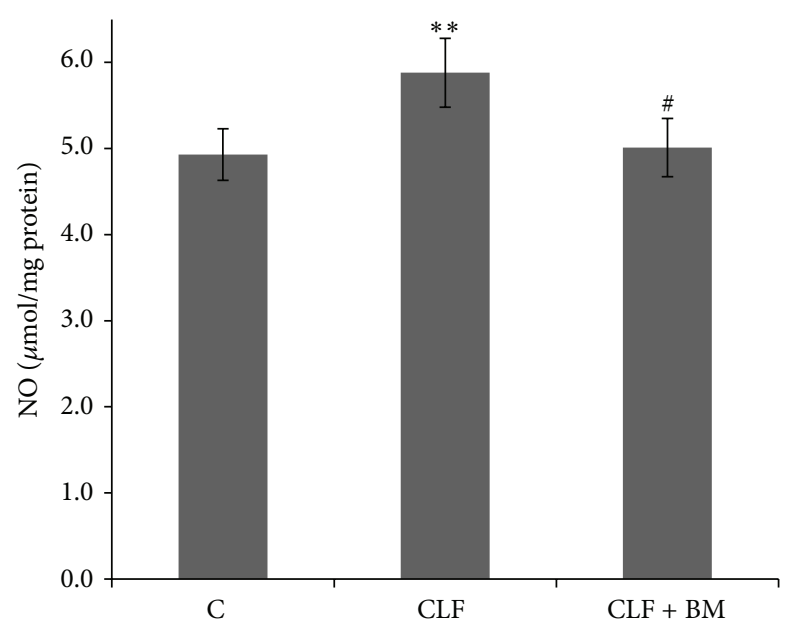

(c)

FIGURE 2: Expression profile of nNOS ((a) and (b)) and NO level (c) in cerebellum of the CLF and BM extract treated CLF rats. Western blot analysis of nNOS was performed as described in the text of methods. In panel (b), normalized densitometric values of nNOS/ $\beta$-actin have been presented as mean \pm SD from three western blot repeats. ${ }^{* *} P<0.01,{ }^{* * *} P<0.001$; (control versus CLF rats) ${ }^{\#} P<0.05$, ${ }^{\# \#} P<0.001$; (CLF versus CLF + BM rats). C: control, CLF: chronic liver failure, CLF + BM: chronic liver failure + Bacopa monnieri extract.

greater susceptibility to undergo oxidative and nitrosative stress during HA and CLF led excitotoxicity $[12,13]$.

It has been described that lesion in cerebellum impairs acquisition of memory consolidation and deranges motor functions $[10,11]$. The CLF rats used in this experiment have recently been reported to show cognitive impairment and deficit in motor functions as well [2]. Since such neurochemical alterations leading to neurobehavioural changes are known to emanate from abnormal NMDAR activity [1], it is argued that a shift from a NR2A combination to a NR2B combination of NMDAR in the cerebellum of the CLF rats might be accountable for developing $\mathrm{HE}$ associated symptoms observed in these CLF rats [2]. Moreover, keeping aside these explanations, the findings provided a basis to alter NMDAR constitution, instead of shutting this ion-channel off, as a therapeutic option to bring recovery from the HE symptoms.
To test this hypothesis, BM extract was chosen due to the two reasons. Firstly, amidst scarcity of the safer neuropharmacological agents, this plant extract is demonstrated to improve neuronal functions by modulating brain chemistry in many ways [28]. Secondly, BM is now evident to modulate activity of the neurotransmitter receptors like serotonergic receptors during epilepsy [29] and dopaminergic and cholinergic signaling in Parkinson's and Alzheimer's diseases [30, 31]. However, report is limited on modulation of NMDA receptor activity by BM. We observed a remarkable shift from a declined ratio of NR2A/NR2B (neurodegeneration supportive combination) in the cerebellum of CLF rats towards their normal level when these CLF rats were administered with BM extract (Figure 1(e)). This finding suggested that $\mathrm{BM}$ is able to alter constitution of the functional NMDAR tetramer by differential expression of the two glutamate binding subunits in cerebellum of the CLF rats. Indeed, in 


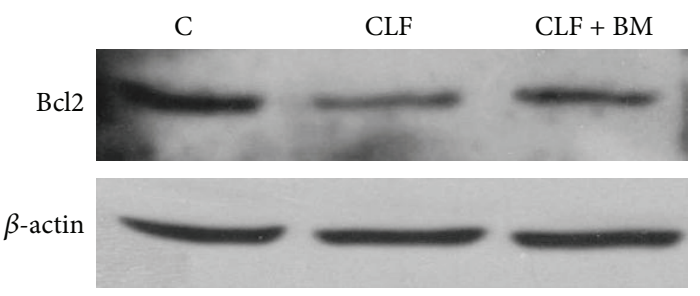

(a)

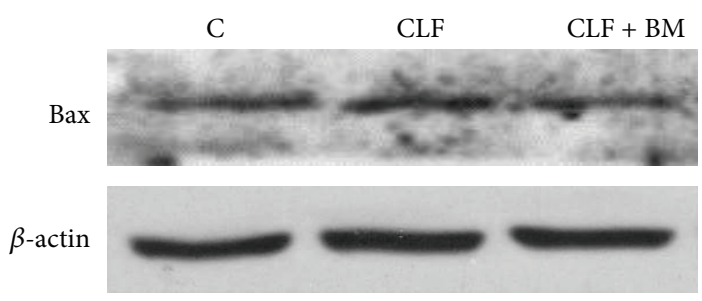

(c)

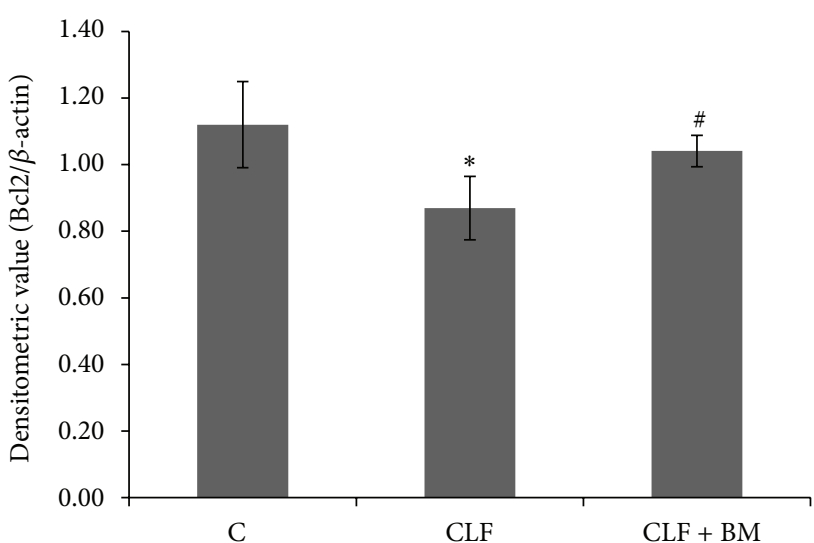

(b)

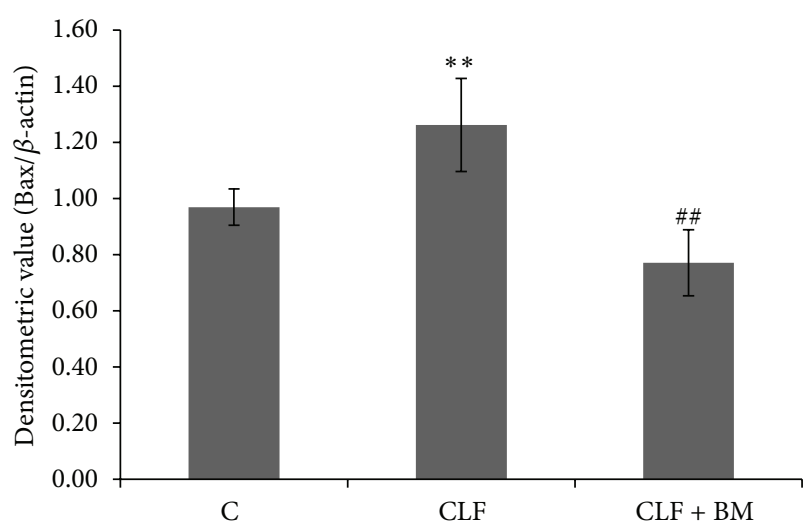

(d)

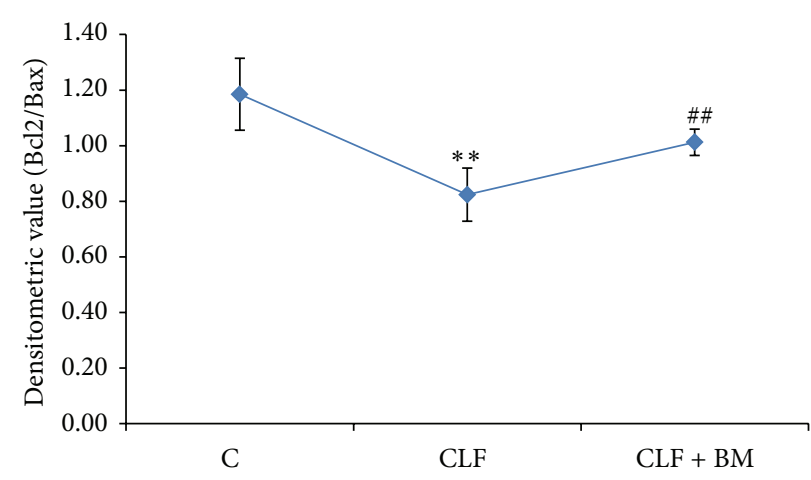

(e)

Figure 3: Expression profile of Bcl2 ((a) and (b)) and Bax ((c) and (d)) in cerebellum of the CLF and BM extract treated CLF rats. Western blot analysis of $\mathrm{Bcl} 2$ and Bax was performed as described in the text of methods. The level of $\beta$-actin was probed as the loading control. In panels (b), (d), and (e), normalized densitometric values of $\mathrm{Bcl} 2 / \beta$-actin, $\mathrm{Bax} / \beta$-actin, and $\mathrm{Bcl} 2 / \mathrm{Bax}$ ratio, respectively, have been presented as mean \pm SD from three western blot repeats. ${ }^{*} P<0.05,{ }^{* *} P<0.01$; (control versus CLF rats) ${ }^{\#} P<0.05$, ${ }^{\#} P<0.01$; (CLF versus CLF + $\mathrm{BM}$ rats). C: control, CLF: chronic liver failure, CLF + BM: chronic liver failure + Bacopa monnieri extract.

an epilepsy model, it has been demonstrated that BM could alter NR1 gene expression [32], thus suggesting BM as a modulator of NMDAR subunit expression. A similar finding on modulation of NR1 by BM was reported in a hypoxia model also [42]. Importantly, such a change in NR1 expression was found accountable for modulating glutamatergic signaling in cerebellum of those rats [32]. Thus, it is argued that shifting from a neurodegenerative NR2B overexpression towards the neuroprotective NR2A level due to the treatment with BME (Figure 1) could also account for preventing downstream undesirable neurochemical changes in cerebellum of the CLF rats.

In a number of excitotoxic models, including CLF led HE, nNOS activation is considered as the most common event after NMDAR activation [13, 43]. It is initiated by influx of $\mathrm{Ca}^{2+}$ which overactivates this enzyme to produce excess 
$\mathrm{NO}$ in the postsynaptic neurons [44]. NO is a molecule of pleiotropic effects; however, when produced in excess in the brain cells, it uses more than one mechanism to induce neuronal dysfunction [20,21]. A milder increase in NO level is likely to initiate mitochondrial dysfunction led neuronal apoptosis. Ratio of $\mathrm{Bcl} 2$ versus $\mathrm{Bax}$ is considered as the most effective regulators of mitochondrial dysfunction led apoptosis [45] and hence relative levels of both these proteins are considered as a reliable tool to assay whether a cell is likely to undergo internal apoptotic process [46]. In cerebellum of the CLF rats, a significantly increased level of nNOS coincides well with a similar increment in NO level (Figure 2). This is consistent with a significant decline in the $\mathrm{Bcl} 2 / \mathrm{Bax}$ ratio (Figure 3). Moreover, all these factors were found to be reversed to regain their normal levels in the cerebellum when those CLF rats were treated with BM extract (Figures 1-3). Recent studies have also shown that BM extract downregulates $\mathrm{Bax}$ and upregulates $\mathrm{Bcl} 2$ and thus provides neuroprotection in several neurological disease models [47]. Thus, the findings of Figures 1-3 together advocate for concordant modulation of NMDA receptor constitution and nNOS led apoptotic activation by ethanolic extract of BM (CDRI-08) in cerebellum of the CLF rats.

\section{Conclusion}

Opposing roles of the two main glutamate binding subunits, NR2A and NR2B, of NMDA receptor advocate for modulation of NMDAR constitution, as an effective mechanism to normalize its excitotoxic effects without blocking its ionchannel activity. During recent past, studies on efficacy of Bacopa monnieri extract have been shown to prevent neurodegenerative diseases by modulating neurochemistry of the brain cells, but with little information about modulation of NMDAR overactivation led excitotoxicity. The present findings demonstrate that $\mathrm{BM}$ extract is able to modulate NMDA receptor signaling by bringing reciprocal changes in the expression of its two glutamate binding subunits, NR2A and NR2B, and thus provide a novel approach to normalize NMDAR overactivation effects without blocking this ion channel.

\section{Conflict of Interests}

The authors declare no conflict of interests.

\section{Acknowledgments}

The work was financially supported by DBT Grant (BT/ PR14049/Med/30/344/2010) to Surendra Kumar Trigun and the award of CSIR SRF to Papia Mondal. The facilities of DST-FIST and UGC-CAS programs in the Department of Zoology and BHU-DBT ISLS facilities in Faculty of Science are also acknowledged. The authors thank Professor Hemant Singh, Lumen Research Foundation, Chennai, for providing the ethanolic extract of Bacopa monnieri (CDRI-08).

\section{References}

[1] V. Felipo, "Hyperammonemia," in Handbook of Neurochemistry and Molecular Neurobiology, Brain and Spinal Cord Trauma, A. laztha, N. Banik, and S. K. Ray, Eds., pp. 43-69, Springer, Berlin, Germany, 3rd edition, 2009.

[2] S. Singh and S. K. Trigun, "Low grade cirrhosis induces cognitive impairment and motor dysfunction in rats: could be a model for minimal hepatic encephalopathy," Neuroscience Letters, vol. 559, pp. 136-140, 2014.

[3] A. T. Blei, J. Cordoba, and The Practice Parameters Committee of the American College of Gastroenterology, "Hepatic encephalopathy," American Journal of Gastroenterology, vol. 96, pp. 635-643, 2001.

[4] J. S. Bajaj, J. B. Wade, and A. J. Sanyal, "Spectrum of neurocognitive impairment in cirrhosis: implications for the assessment of hepatic encephalopathy," Hepatology, vol. 50, no. 6, pp. 20142021, 2009.

[5] F. F. Poordad, "Review article: the burden of hepatic encephalopathy," Alimentary Pharmacology and Therapeutics, vol. 25, no. 1, pp. 3-9, 2007.

[6] J. S. Bajaj, "Minimal hepatic encephalopathy matters in daily life," World Journal of Gastroenterology, vol. 14, no. 23, pp. 36093615, 2008.

[7] S. Mechtcheriakov, I. W. Graziadei, A. Kugener et al., "Motor dysfunction in patients with liver cirrhosis: impairment of handwriting," Journal of Neurology, vol. 253, no. 3, pp. 349-356, 2006.

[8] S. J. Gilberstadt, H. Gilberstadt, L. Zieve, B. Buegel, R. O. Collier Jr., and C. J. McClain, "Psychomotor performance defects in cirrhotic patients without overt encephalopathy," Archives of Internal Medicine, vol. 140, no. 4, pp. 519-521, 1980.

[9] V. Felipo and R. F. Butterworth, "Neurobiology of ammonia," Progress in Neurobiology, vol. 67, no. 4, pp. 259-279, 2002.

[10] C. C. Gandhi, R. M. Kelly, R. G. Wiley, and T. J. Walsh, "Impaired acquisition of a Morris water maze task following selective destruction of cerebellar purkinje cells with OX7saporin," Behavioural Brain Research, vol. 109, no. 1, pp. 37-47, 2000.

[11] M. G. Leggio, P. Neri, A. Graziano, L. Mandolesi, M. Molinari, and L. Petrosini, "Cerebellar contribution to spatial event processing: characterization of procedural learning," Experimental Brain Research, vol. 127, no. 1, pp. 1-11, 1999.

[12] S. Singh, R. K. Koiri, and S. K. Trigun, "Acute and chronic hyperammonemia modulate antioxidant enzymes differently in cerebral cortex and cerebellum," Neurochemical Research, vol. 33, no. 1, pp. 103-113, 2008.

[13] S. Singh and S. K. Trigun, "Activation of neuronal nitric oxide synthase in cerebellum of chronic hepatic encephalopathy rats is associated with up-regulation of NADPH-producing pathway," Cerebellum, vol. 9, no. 3, pp. 384-397, 2010.

[14] P. Mondal and S. K. Trigun, "Pannexin1 as a novel cerebral target in pathogenesis of hepatic encephalopathy," Metabolic Brain Disease, vol. 29, no. 4, pp. 1007-1015, 2014.

[15] S. Cull-Candy, S. Brickley, and M. Farrant, "NMDA receptor subunits: diversity, development and disease," Current Opinion in Neurobiology, vol. 11, no. 3, pp. 327-335, 2001.

[16] Y. Liu, P. W. Tak, M. Aarts et al., "NMDA receptor subunits have differential roles in mediating excitotoxic neuronal death both in vitro and in vivo," The Journal of Neuroscience, vol. 27, no. 11, pp. 2846-2857, 2007. 
[17] O. A. Shipton and O. Paulsen, "GluN2A and GluN2B subunitcontaining NMDA receptors in hippocampal plasticity," Philosophical Transactions of the Royal Society B: Biological Sciences, vol. 369, no. 1633, 2014.

[18] C. Montoliu, R. Rodrigo, P. Monfort et al., "Cyclic GMP pathways in hepatic encephalopathy: neurological and therapeutic implications," Metabolic Brain Disease, vol. 25, no. 1, pp. 39-48, 2010.

[19] M. V. Doucet, A. Harkin, and K. K. Dev, “The PSD-95/nNOS complex: new drugs for depression?” Pharmacology \& Therapeutics, vol. 133, no. 2, pp. 218-229, 2012.

[20] H. Prast and A. Philippu, "Nitric oxide as modulator of neuronal function," Progress in Neurobiology, vol. 64, no. 1, pp. 51-68, 2001.

[21] F. X. Guix, I. Uribesalgo, M. Coma, and F. J. Muñoz, "The physiology and pathophysiology of nitric oxide in the brain," Progress in Neurobiology, vol. 76, no. 2, pp. 126-152, 2005.

[22] E. A. Waxman and D. R. Lynch, "N-methyl-D-aspartate receptor subtypes: multiple roles in excitotoxicity and neurological disease," Neuroscientist, vol. 11, no. 1, pp. 37-49, 2005.

[23] V. Felipo, "Contribution of altered signal transduction associated to glutamate receptors in brain to the neurological alterations of hepatic encephalopathy," World Journal of Gastroenterology, vol. 12, no. 48, pp. 7737-7743, 2006.

[24] M. Llansola, R. Rodrigo, P. Monfort et al., "NMDA receptors in hyperammonemia and hepatic encephalopathy," Metabolic Brain Disease, vol. 22, no. 3-4, pp. 321-335, 2007.

[25] S. A. Lipton and P. A. Rosenberg, "Mechanisms of disease: excitatory amino acids as a final common pathway for neurologic disorders," The New England Journal of Medicine, vol. 330, no. 9, pp. 613-622, 1994.

[26] D. R. Lynch and R. P. Guttmann, "Excitotoxicity: perspectives based on N-methyl-D-aspartate receptor subtypes," Journal of Pharmacology and Experimental Therapeutics, vol. 300, no. 3, pp. 717-723, 2002.

[27] C. Ikonomidou and L. Turski, "Why did NMDA receptor antagonists fail clinical trials for stroke and traumatic brain injury?” The Lancet Neurology, vol. 1, no. 6, pp. 383-386, 2002.

[28] K. J. Gohil and J. J. Patel, "A review on Bacopa monniera: current research and future prospects," International Journal of Green Pharmacy, vol. 4, no. 1, pp. 1-9, 2010.

[29] A. Krishnakumar, P. M. Abraham, J. Paul, and C. S. Paulose, "Down-regulation of cerebellar 5-HT2C receptors in pilocarpine-induced epilepsy in rats: therapeutic role of Bacopa monnieri extract," Journal of the Neurological Sciences, vol. 284, no. 1-2, pp. 124-128, 2009.

[30] M. Singh, V. Murthy, and C. Ramassamy, "Neuroprotective mechanisms of the standardized extract of Bacopa monniera in a paraquat/diquat-mediated acute toxicity," Neurochemistry International, vol. 62, no. 5, pp. 530-539, 2013.

[31] N. Uabundit, J. Wattanathorn, S. Mucimapura, and K. Ingkaninan, "Cognitive enhancement and neuroprotective effects of Bacopa monnieri in Alzheimer's disease model," Journal of Ethnopharmacology, vol. 127, no. 1, pp. 26-31, 2010.

[32] R. Khan, A. Krishnakumar, and C. S. Paulose, "Decreased glutamate receptor binding and NMDA R1 gene expression in hippocampus of pilocarpine-induced epileptic rats: neuroprotective role of Bacopa monnieri extract," Epilepsy and Behavior, vol. 12, no. 1, pp. 54-60, 2008.

[33] O. H. Lowry, N. J. Rosebrough, A. L. Farr, and R. J. Randall, "Protein measurement with the Folin phenol reagent," The Journal of Biological Chemistry, vol. 193, no. 1, pp. 265-275, 1951.
[34] K. V. H. Sastry, R. P. Moudgal, J. Mohan, J. S. Tyagi, and G. S. Rao, "Spectrophotometric determination of serum nitrite and nitrate by copper-cadmium alloy," Analytical Biochemistry, vol. 306, no. 1, pp. 79-82, 2002.

[35] D. R. Lynch and R. P. Guttmann, "NMDA receptor pharmacology: perspectives from molecular biology," Current Drug Targets, vol. 2, no. 3, pp. 215-231, 2001.

[36] L. V. Kalia, S. K. Kalia, and M. W. Salter, "NMDA receptors in clinical neurology: excitatory times ahead," The Lancet Neurology, vol. 7, no. 8, pp. 742-755, 2008.

[37] A. Lau and M. Tymianski, "Glutamate receptors, neurotoxicity and neurodegeneration," Pflugers Archiv European Journal of Physiology, vol. 460, no. 2, pp. 525-542, 2010.

[38] M. M. Zeron, O. Hansson, N. Chen et al., "Increased sensitivity to $\mathrm{N}$-methyl-D-aspartate receptor-mediated excitotoxicity in a mouse model of Huntington's disease," Neuron, vol. 33, no. 6, pp. 849-860, 2002.

[39] A. J. Williams, J. R. Dave, X. M. Lu, G. Ling, and F. C. Tortella, "Selective NR2B NMDA receptor antagonists are protective against staurosporine-induced apoptosis," European Journal of Pharmacology, vol. 452, no. 1, pp. 135-136, 2002.

[40] M. Chen, T.-J. Lu, X.-J. Chen et al., "Differential roles of NMDA receptor subtypes in ischemic neuronal cell death and ischemic tolerance," Stroke, vol. 39, no. 11, pp. 3042-3048, 2008.

[41] K. Erreger, S. M. Dravid, T. G. Banke, D. J. A. Wyllie, and S. F. Traynelis, "Subunit-specific gating controls rat NR1/NR2A and NR1/NR2B NMDA channel kinetics and synaptic signalling profiles," The Journal of Physiology, vol. 563, no. 2, pp. 345-358, 2005.

[42] C. S. Paulose, F. Chathu, S. Reas Khan, and A. Krishnakumar, "Neuroprotective role of Bacopa monnieri extract in epilepsy and effect of glucose supplementation during hypoxia: glutamate receptor gene expression," Neurochemical Research, vol. 33, no. 9, pp. 1663-1671, 2008.

[43] V. L. R. Rao, R. M. Audet, and R. F. Butterworth, "Increased nitric oxide synthase activities and $\mathrm{L}-\left[{ }^{3} \mathrm{H}\right]$ arginine uptake in brain following portacaval anastomosis," Journal of Neurochemistry, vol. 65, no. 2, pp. 677-681, 1995.

[44] P. Monfort, M.-D. Muñoz, A. ElAyadi, E. Kosenko, and V. Felipo, "Effects of hyperammonemia and liver failure on glutamatergic neurotransmission," Metabolic Brain Disease, vol. 17, no. 4, pp. 237-250, 2002.

[45] C. M. Snyder, E. H. Shroff, J. Liu, and N. S. Chandel, "Nitric oxide induces cell death by regulating anti-apoptotic $\mathrm{Bcl} 2$ family members," PLoS ONE, vol. 4, no. 9, Article ID e7059, 2009.

[46] R. K. Koiri and S. K. Trigun, "Dimethyl sulfoxide activates tumor necrosis factor $\alpha$-p53 mediated apoptosis and down regulates $\mathrm{D}$-fructose-6-phosphate-2-kinase and lactate dehydrogenase-5 in Dalton's Lymphoma in vivo," Leukemia Research, vol. 35, no. 7, pp. 950-956, 2011.

[47] R. B. Thomas, S. Joy, M. S. Ajayan, and C. S. Paulose, "Neuroprotective potential of bacopa monnieri and bacoside a against dopamine receptor dysfunction in the cerebral cortex of neonatal hypoglycaemic rats," Cellular and Molecular Neurobiology, vol. 33, no. 8, pp. 1065-1074, 2013. 


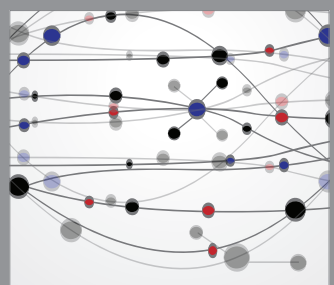

The Scientific World Journal
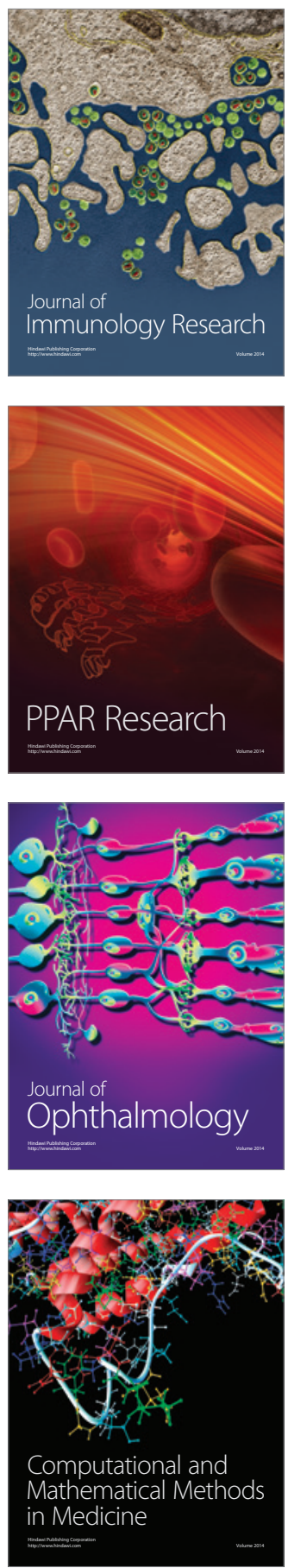

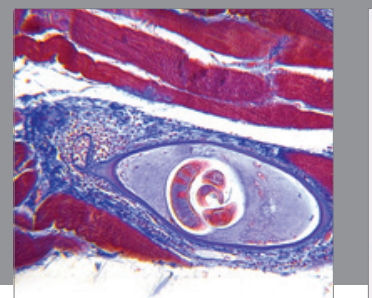

Gastroenterology

Research and Practice
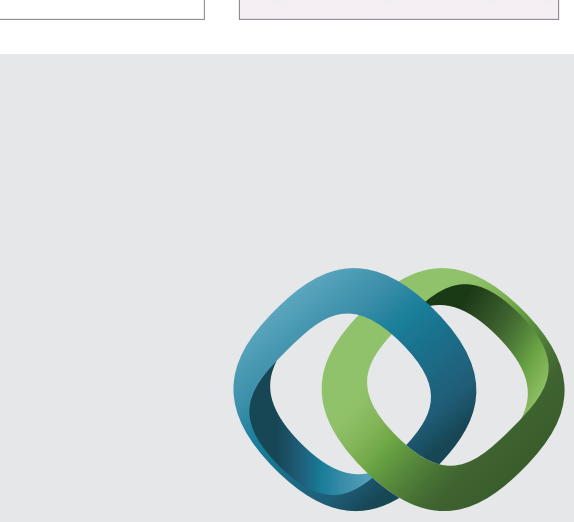

\section{Hindawi}

Submit your manuscripts at

http://www.hindawi.com
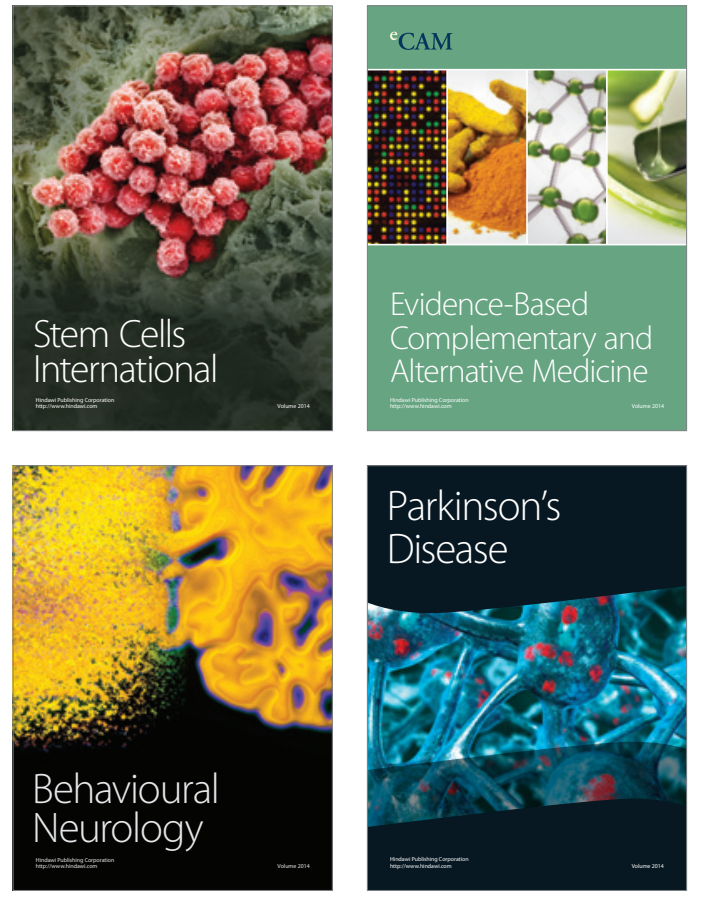
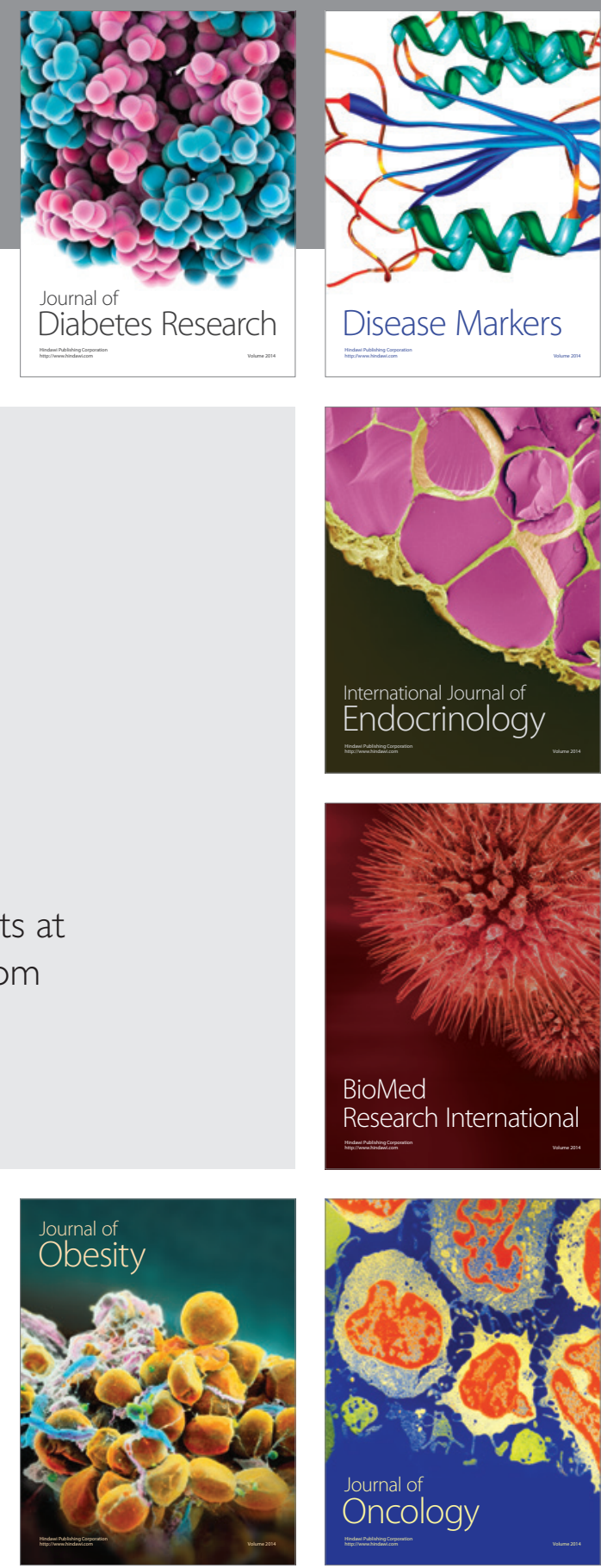

Disease Markers
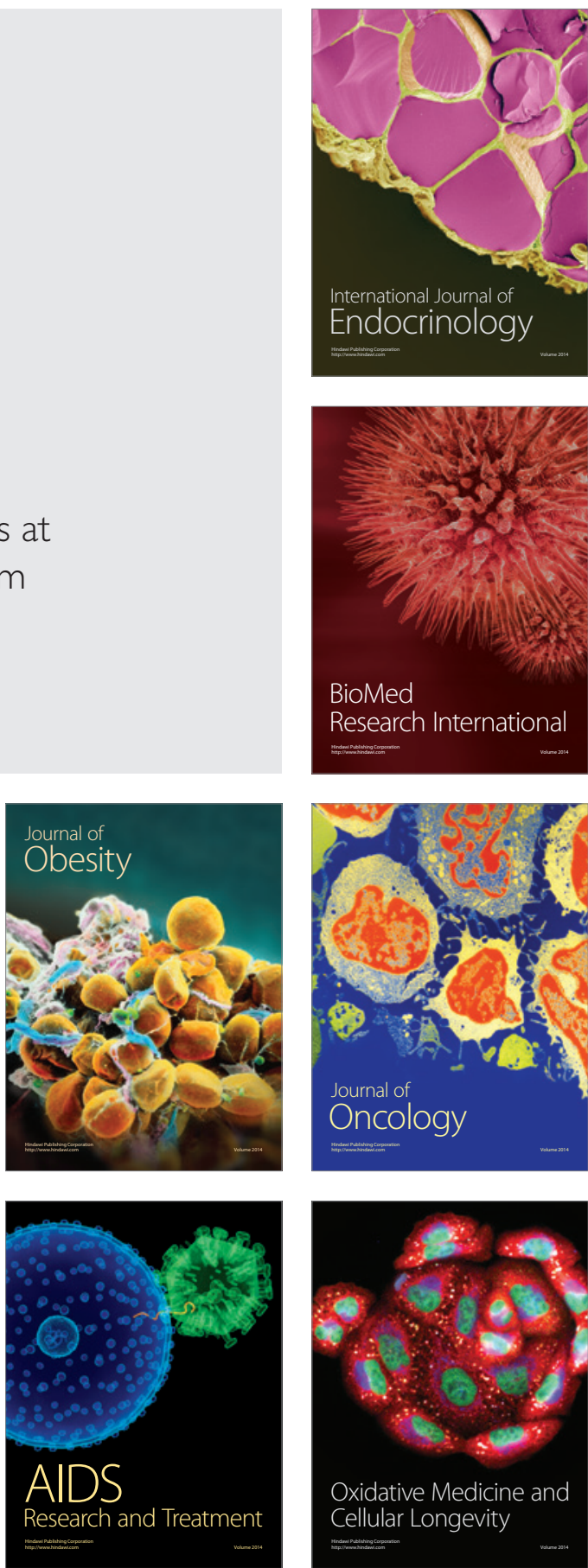